\title{
Increasing of the Work Productivity of CMM Machine by Applying of Augmented Reality Technology
}

\author{
Jozef Torok, Marek Kocisko, Monika Teliskova ${ }^{a}$ and Miroslav Janak \\ Technical University of Kosice, Faculty of Manufacturing Technologies with a Seat in Presov, Sturova 31, 08001 Presov, Slovak Republic
}

\begin{abstract}
This article is focused on the area of metrology and possibility of connection between 3D coordinate measuring machine and augmented reality technology. The aim of this contribution was to bring close the possibilities of augmented reality for example by visualization of important zones during the measurement process. The use of this application is very useful and if the user needs to measure some object after the long time, he does not know, which points were defined for alignment or which position was set for measured object. In this case the use of augmented reality can be very useful.
\end{abstract}

\section{Introduction}

The main aim of all companies is to produce profit and for that every company tries to use the contained technology more effective to ensure minimization of production costs of their goods. This situation forces the companies to look for a solution to all steps of production process and also to output control by searching of defects on own products. One possibility for precise check is usage of coordinate measuring machines. They can relatively quickly and reliably detect the necessary dimensions and document compliance of production with manufacturing documentation. Nowadays, for example the automotive industry without such controls we cannot imagine. This group of modern coordinate measuring technique offers the universal usage in control of the various shaped parts. Universality of Coordinate Measuring Machine in short CMM is in accurate measurement on single machine be measuring length dimensions, angles, evaluation of form and location tolerances.

\section{Coordinate measuring machine and measurement process}

The Faculty of Manufacturing Technologies of Technical University of Kosice with a seat in Presov uses 3D measuring machine Rapid from company Thome Praezision. [10] This machine is equipped with a precise optimized conduction of granite and its thermal stability and high precision of conduction ensure the accurate measurement results without putting the software compensation. The conduct tracks are protected by flexible cover of accordion type. [10]

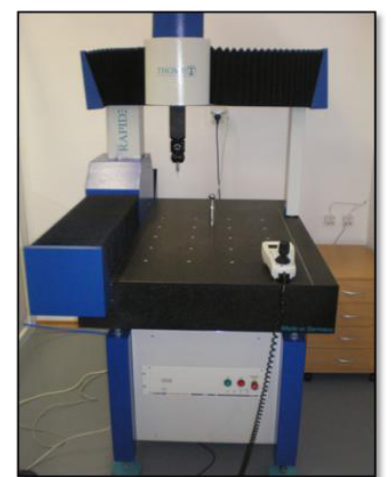

Figure 1. 3D CMM - Thome Praezision rapid.

Each measured object is composed of a number of elements, i.e. theoretical elements such as point, plane, cylinder or sphere in which it is possible to evaluate the several parameters - characteristics. These characteristics may describe one element, for example radius, length, flatness, etc., or may define the relationship between elements. The one element can detect several characteristics, e.g. the circle's diameter, coordinates of the center, roundness,... or the several elements can detect one common characteristic, e.g. angle between the planes or perpendicularity. CMM's can identify the geometry of the measured object by specifying the spatial coordinates of several points of the object. By usage of their information's can then be determined the replacement of object geometry [10].

The first necessary step in the measurement process is always drawing up of measurement process plan. It contains of documentation, clamping method of object, clamping products, etc.... The measurement plan should include a measurement strategy, from which must be

\footnotetext{
a Corresponding author: monika.teliskova@tuke.sk
} 
clear the requirement on the accuracy of measurement and the optimal procedure for recognition of possible disparities 7 .

Before each measurement user has to define a coordinate system which is usually somehow relevant to the measured object. By the initial definition of measurement process is always automatically unpacked menu with possibilities of what a user wants to measure, define or construct. Each measured object has a different dimensions and different shape. In the measurement process is necessary to determine what will be measured. During the measurement the measuring head moves towards the object and in the moment when it touches the object at the required point sensor sends an electrical signal with the information of coordinates of a point on object's surface. The measuring head is controlled manually by joystick or can be controlled by the computer (CNC mode).

It is necessary to determine the minimum number of points for definition of this element. For example to measure a circle it is needed to set at least three points but for each geometrical element it is required to set a different number of points depending on the required accuracy. For the most important and used geometrical features are available automatically categories of scanning (point, line, circle, radius, plane, sphere, cone, torus, flat points, cut, rectangle, groove,...).

From the measurement results can be calculated the angle, distance, cutting elements, symmetry,.... There are also shape and position tolerances as: straightness, flatness, roundness, cylindricity, surface shape, parallelism, perpendicularity, angularity, position, coaxiality, concentricity, symmetry, and runout.

\subsection{Program}

Creation of programs for inspection of components is currently very used mainly for control in mass production of components where individual measurement without some kid of automation would be very time-consuming. It is necessary to set the essential parameters for the correct measurement and then create a program. The basic steps to a good program are basically always the same. In the initial phase before the creation of the program is necessary to define the nature of probe - its diameter and all positions of rotation of measuring head and after that all these positions need to be calibrated.

At the beginning of the measurement it is necessary to define the coordinate system of the measured object. They can be used various methods of alignment: 1 point, geometric alignment, alignment on 6 points, with 3 centres, on the plane and 2 centres, Best-Fit and RPS,..., but the most common is 3-2-1 alignment.

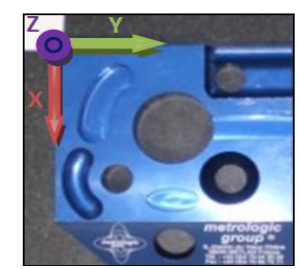

Figure 2. The measured object with the created coordinate system
The first measured object is fixed on the granite table, user defines the object coordinate system - alignment and measures the necessary elements on the object. This first measurement is performed as a classical measure where the user manually selects the element and measures by measuring probe. In the measuring process is very important to define detour points to avoid later the collisions in the automatic measuring process. They are set during the measurement of elements, but also during the move of probe from one element to another. Measurement of this first object is then repeated using a CNC program.

The following objects for sensing are fixed on the granite table of CMM and measurement is done by $\mathrm{CNC}$ program (Fig. 3), but at the beginning of each new measurement cycle the needed points for alignment are defined manually by user. The method is advantageous because the user can measure whole series of identical objects.

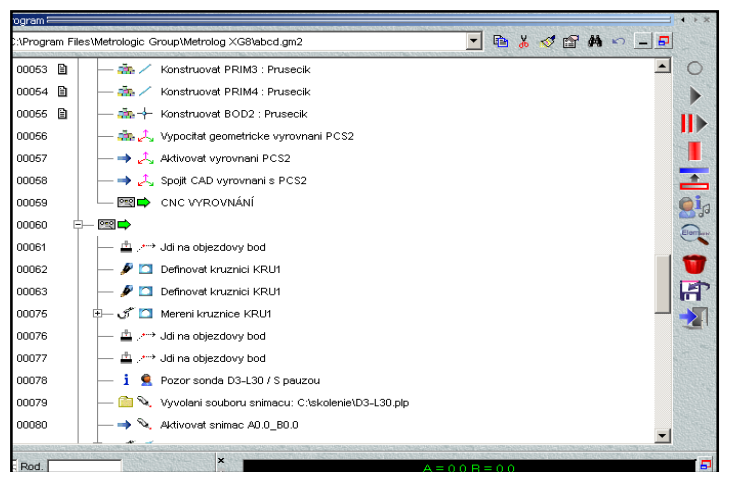

Figure 3. The part of the program for measuring

\subsection{Augmented reality and its use in the metrology of CMM}

Augmented reality (in short AR) is a live direct or indirect realistic view of the world with augmented elements generated by a computer. The presentation of reality can by enriched with digital elements like images text or nowadays more often by models of objects.

The camera, which is connected to a computing core, senses the fiducially marker (structured image) and special application detects the shooting scene, then places on the image supplementary information (objects, text, video,...) or can launch the pre-programmed actions in real time. For the detection of the object can be used also edge tracking technology which in some cases can be more precise.

The application is trying to identify a marker or object to determine its position and orientation in space of scene. Based on input information the application can add 3D model to the augmented scene at the right place and in the correct orientation. Some software can also "learn" to perform actions for example by tracking user's hands or face (so called face tracking). These gestures can then be used to run the programmed actions and so to perform sort of application control (proper positioning of the scene).

Correct recognition of marker depends on the quality and resolution of the camera by the right lighting on scene and graphical look of marker. Therefore very often 
are used simple black and white icons (symbols, QR codes, etc.).

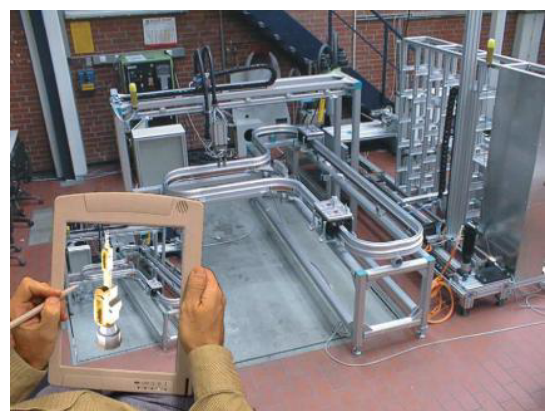

Figure 4. Modeling of new production systems by using AR solutions elements.

At our Faculty of Manufacturing Technologies with a seat in Presov we use the augmented reality as a support technology in assembling processes applications for example as in the paper "Open Source Tools in Assembling Process Enriched with elements of Augmented Reality" 2 The authors describe the current solutions in these field of AR and represent their own solutions to the position and orientation tracking and move of virtual objects by the equipment working not only on the marker principle. In this article are described Open Source software applications combinated with special rotating position table which connects the proper sensor data and virtual augmented environment elements. Contribution offers some examples of application of augmented reality by hand layering of composite fabrics (Fig. 5) and assembly process (Fig. 6) .
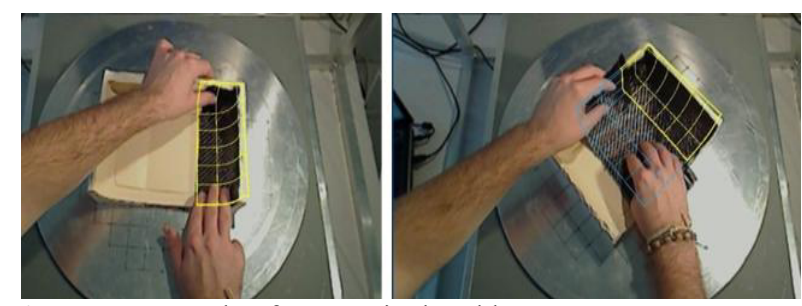

Figure 5. Example of composite hand lay - up process

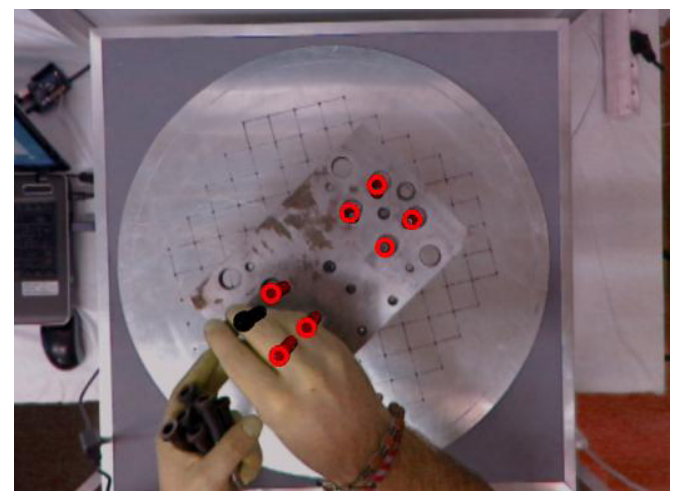

Figure 6. Realization of assembly activities with displaying of inserted parts

One of new trends in the field of metrology is the effort to involve a wider basis of variety specialized techniques suitable for the process of dimensional control of objects where the variety of included devices can simplify and facilitate the control process.
One example is hybrid contact-optical coordinate system, which was specially designed for measuring and analysis of an object geometry. This system combines the accuracy of contact measurement regarding the identification of measurement points and the speed of full-field structured light optical scanning methods. The measurement process starts from optical acquisition by structured light system positioned around the working space and the numerical analysis where is a set of surface points calculated which should be finally re-measured by the CMM. Both set-ups work in a common coordinate system created according to existing metrological strategies. Final metrological analysis is performed using classic certified metrological software. The mentioned article describes also the maximum permissible error of length measurement of the hybrid system 8 .

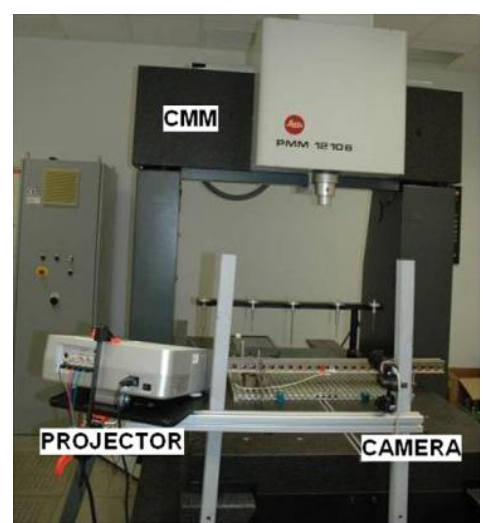

Figure 7. Laboratory of hybrid system

The system can use more common CCD cameras for calibration so the paper 1 evaluates the accuracy of different calibrations and measurement methods used in 3D stereo vision with CCD cameras. This article describes several precision tests for different methods, determining their error limits under specified conditions of operation. Two practical applications are described as cost-effective system for the measurement of free-form surfaces able to generate CAD models and measuring programs for CMMs. The system aims to reduce some difficulties associated with stereo vision and to speed up the traditional digitizing process. The other application involves larger car frame measurement where new automatic measuring system has been developed which allows contactless car frame measurement through two rotating CCD cameras. The more detailed description is presented in 1 .

Virtual reality technology in metrology is nowadays mainly used in the simulation of measurement process and for control of potential collision situations of measuring probe and sensed object caused by operator, simulation of hardly reachable access to the required elements of object,...

The most common measuring softwares for CMM offers the possibility of offline programming of CMM, some applications enable the simulation of CMM and for example a human operator or automated system for loading and unloading the measured object. These facts are described in article 6 . The paper presents the features and advantages offered by Delmia V5 for simulating a 
complete measuring environment encompassing: the CMM, the human operator, a robot or automated system for manipulating the parts.

\section{The possibility of connection augmented reality and 3D measurement machine}

For demonstration of dimensional control and virtual reality technology connection was created real example, which uses 3D CMM Thome Rapid, metrology software Metrolog XG and software based on open source philosophy Blender, for augmented reality. For sensing of real world a classical web camera attached to the monitor was used.

For test measurement a proven component with few base shapes was chosen to provide necessary examples. By on-line programming the CNC program was created to measure the required dimensions. Without the use of the AR technology the program after start required six points for manual definition of object surface and then the automatic control process could start.

For concrete objects can be created applications which are prepared to show position of needed points not only for basic alignment. The real world is captured by the camera mounted on the back of the monitor which shows the augmented scene. So the user sees exactly how the real object is placed on the measuring table and the needed points are shown in order how the user need to define them on the object. The same effect can be provided with usage of HMD glasses.

On the start of the application camera senses the position of the marks, information about location of object placed near of the measured object important for the computing core and so in the next step (in real time) are generated virtual augmented objects for needed points. In this presented solution the measured object must be always placed in the relative same location respect to the marks placed on the table according to proper visualization of needed points. For the calibration process it would be good to use the preparation holes in the measuring table but there may cause confusion because they are same size and placed in regular intervals. Nowadays for faster and more proper placement recognition of measured product team of faculty works on advancer edge based tracking system.

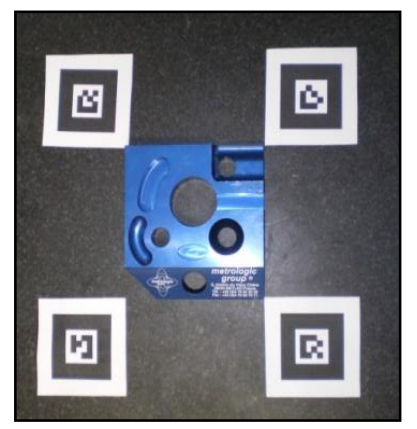

Figure 8. Location of object and calibration marks

Measured object should be placed in an exact fixture which ensures always the same position of the object. If the user measures without the fixtures the calibration marks is needed to place the object in exact location. An example of such a placement is shown in Fig. 10. The component is placed in a position where its three corners are touching the corners of calibration marks. It ensures always the same position of the measured object to the calibration marks so the user gets the correct delineation of the position of the required points needed for alignment of object and so for follow-up measurement.

After the detection of objects location the application shows needed surfaces for the measurement. The augmented surfaces are marked by red colour, three points up on the surface - to define the plane of which is defining the z-axis, 2 points on the side surface - to define a straight line, of which goes the $y$ axis and the last point on the back surface which defines the last coordinate $-\mathrm{x}$ axis. This information together defines the coordinate system of the object. Application shows the position of all six points and then displays the animation of probe path to needed positions with defined points from first to the final point.

Fig. 9 shows running application and the calibration of object position by finished six needed points for alignment of the object.
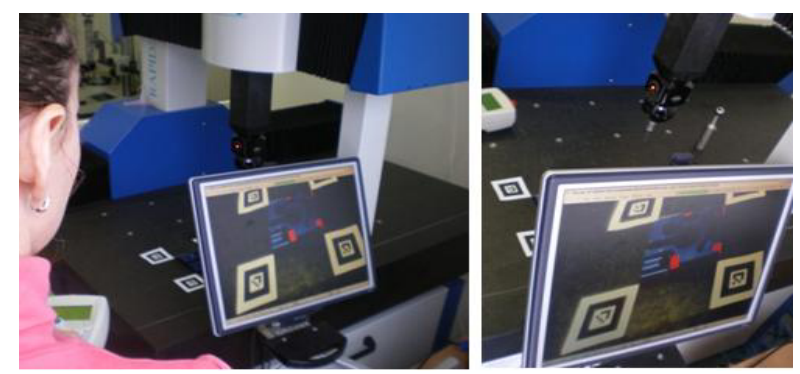

Figure 9. Augmented points needed for aligment of measured object

Fig. 10 illustrates the animation of required points for alignment. On the monitor user sees the real world with added AR application. This application shows him where he needs to define the alignment points. On the monitor screen user sees the real and augmented virtual measuring probe. If the user confirms some points software automatically will show the next points needed to measure.
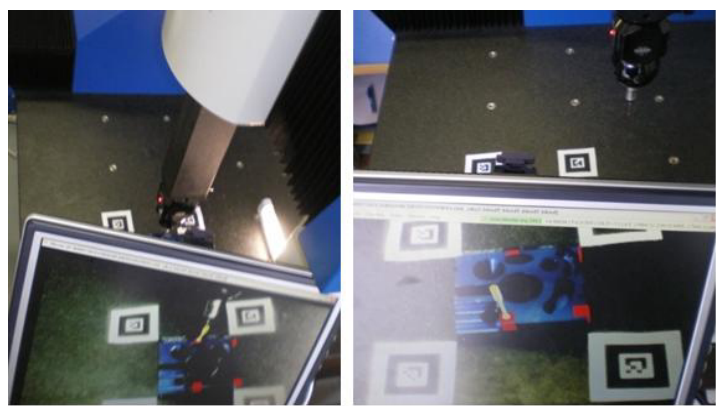

Figure 10. Example of sequential definition of required points

The operator according to the animation defines points and immediately after their definition he can start the automatic process of control which is already done without the operator assistance. The operator only is overseeing the measurement where he can change the 
speed of the measurement or to stop the process. If the program is correct designed and the object is correct clamped with well defined points during the measurement process shouldn't occur no collisions with the measuring object and measuring probe. Working operator and automatic measurement process on object by CNC program is shown in Fig. 11.
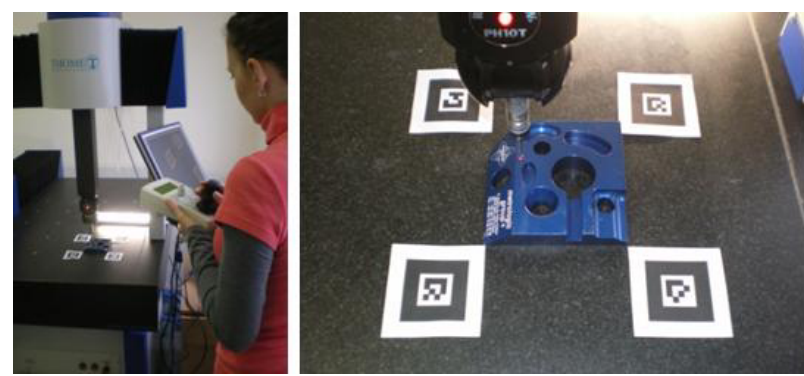

Figure 11. The operator works with the AR application in the measurement process and the automatic measuring of object

By the automatic measurement process we can use augmented reality to show the required values of actual measured dimensions. Fig. 12 shows the measurement of plane and AR application displays the estimated value of flatness for the measured plane according the drawing. Another picture shows the measurement of a circle. The monitor visualizes the real world with application which shows the value of diameter. This application can be used for animation of all important measured dimensions.
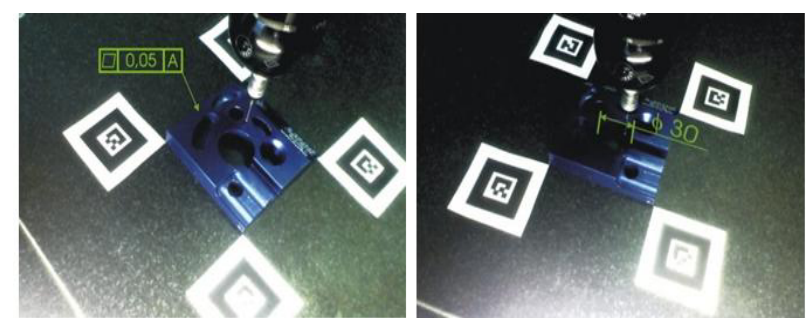

Figure 12. The measuring of flatness and diameter and their visualization with the AR application

\section{Consolution}

This article is focused on the possibility of improving the programming of CMM by involving augmented reality technology in the control process. Its main aim was to illustrate the points needed for alignment of measured object. If the user wants to measure old objects after a long time he does not know where to define the points, how he created the alignment and important surfaces. This application can be wary helpful not only for novices but also for advanced workers. It shows him where and in what order it is necessary to define these points for alignment and correct fast measurement.

The application during the control process shows the value of actually measured element according the drawing and can provide additional informations as room temperature or date about used probe. During the automatic measuring process the user does not need to follow the drawing but the application displays the values of the currently measured element step by step. This application for CMM was developed at the Faculty of Manufacturing Technologies with seat in Presov in Slovakia.

\section{Acknowledgments}

Ministry of Education, Science, Research and Sport of SR supported this work - ITMS project 26220220125.

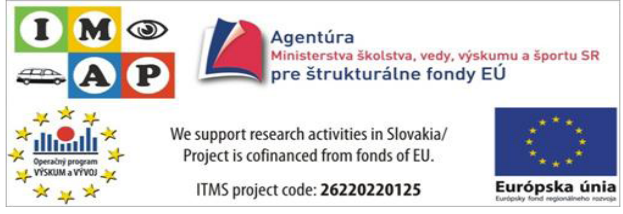

\section{References}

1. J.J. Aguilar, F. Torres, M.A. Lope, Stereo vision for 3D measurement: Accuracy analysis, calibration and industrial applications, Measurement, 18, 4, 193-200 (1996)

2. J. Barna, J. Novak-Marcincin, M. Janak, L. Novakova-Marcincinova, V. Fecova, J. Torok, Open source tools in assembling process enriched with lements of augmented reality, In: VRIC'12: Virtual Reality International Conference, Laval, France, New York: ACM, 2012, 1-8

3. W. Dangelmaier, M. Fischer, J. Gausemeier, M. Grafe, C. Matysczok, B. Mueck, Virtual and augmented reality support for discrete manufacturing system simulation, Computers in Industry, 56, 371383 (2005)

4. J. Dillinger, et al., Moderní strojírenství pro školu i praxy, Praha: Europa -Sobotáles cz. s.r.o., 2007

5. A. Nafi, J.R.R. Mayer, A. Wozniak, Reduced configuration set for the multi-step method applied to machine and probe error separation on a CM, Measurement, 45, 2321- 2329 (2012)

6. A. Nemtu, D. Hurgoiu, S. Poposcu, M. Dragomir, H. Osanna, Training in coordinate measurement using 3D virtual Instruments, Measurement, 45, 2346-2358 (2012)

7. J. Novak-Marcincin, V. Fecova, J. Barna, Dimension control by CMM, In: Quality and Innovation in Engineering and Management, Cluj-Napoca: Technical University, 2011, 147-150

8. J. Sladek, P.M. Blaszczyk, M. Kupiec, R. Sitnik, The hybrid contact-optical coordinate measuring system, Measurement, 44, 503-510 (2011)

9. K. Wendt, M. Franke, F. Härtig, Measuring large 3D structures using four portable tracking laser interferometers, Measurement, 45, 2339-2345 (2012)

10. T. Präzision. http://www.thomepraezision.cz/1200_700_500.html 